\title{
Delayed Intratumoral Hemorrhage after Drug-Eluting Bead Transarterial Chemoembolization for Hepatocellular Carcinoma
}

\author{
Kazue Shiozawa Manabu Watanabe Takashi Ikehara Yu Ogino \\ Tomoko Umakoshi Yasushi Matsukiyo Michio Kogame Teppei Matsui \\ Yoshinori Kikuchi Yoshinori Igarashi Yasukiyo Sumino \\ Division of Gastroenterology and Hepatology, Department of Internal Medicine, \\ Toho University Medical Center, Omori Hospital, Tokyo, Japan
}

\section{Key Words}

Hepatocellular carcinoma - Intratumoral hemorrhage - Transarterial chemoembolization . Drug-eluting bead $\cdot$ Contrast-enhanced ultrasonography

\begin{abstract}
Transarterial chemoembolization (TACE) using a drug-eluting bead (DEB-TACE) for hepatocellular carcinoma (HCC) is a new treatment method. We report on a case of delayed intratumoral hemorrhage after DEB-TACE. An 81-year-old male with hepatitis C virus-related cirrhosis was diagnosed with a HCC of $35 \mathrm{~mm}$ in diameter in S5 detected by dynamic computed tomography (CT) and contrast-enhanced ultrasonography (CEUS). DEB-TACE with DC Bead ${ }^{\circledR}$ and epirubicin hydrochloride was performed because the patient declined to undergo surgical resection. The treatment was completed, and the course after DEB-TACE was favorable. However, right hypochondriac pain suddenly developed about 1 month after DEB-TACE. Unenhanced CT showed an increase of the tumor diameter and intratumoral high-intensity area, which was not enhanced in the arterial phase. CEUS performed at the time of right hypochondriac pain ( 5 weeks after DEB-TACE) showed nonenhancement of almost the entire tumor in the vascular phase. The cause of the symptom may have been DEB-TACE-associated intratumoral hemorrhage. Tumor hemorrhage has been reported after DEB-TACE with tumors $>5 \mathrm{~cm}$ in diameter, and the tumor locations were subcapsular in all previous reports. There has been no case of a tumor with a diameter $<5 \mathrm{~cm}$ distinct from the subcapsular, as was observed in our patient. Incomplete embolization might be the cause of the intratumoral hemorrhage experienced by this case presenting a few risks. To obtain the
\end{abstract}


Shiozawa et al.: Delayed Intratumoral Hemorrhage after Drug-Eluting Bead Transarterial Chemoembolization for Hepatocellular Carcinoma

therapeutic effect of DEB-TACE while preventing the adverse events, it may be important to understand the characteristics of the beads and to apply the appropriate embolization to each individual case.

(C) 2014 S. Karger AG, Basel

\section{Introduction}

Three types of calibrated microspheres have recently been approved in Japan as embolic agents for hypervascular tumors and arteriovenous malformations. To date, two types of embolic microspheres capable of being loaded with drug have been introduced: DC Bead ${ }^{\circledR}$ (Biocompatibles, Farnham, UK) and HepaSphere ${ }^{\circledR}$ (Biosphere Medical, Roissy CDG Cedex, France) [1]. In a phase II randomized controlled study of transarterial chemoembolization (TACE) using a drug-eluting bead (DEB-TACE) for hepatocellular carcinoma (HCC), the PRECISION-V study [2], the response rate after 6 months was $52 \%$ in the DEB-TACE group and $44 \%$ in the TACE group using a Lipiodol ${ }^{\circledR}$ (Laboratoire Guerbet, Aulnay-Sous-Bois, France)/anticancer drug mixture solution (Lip-TACE), showing no significant difference or superiority of DEB-TACE. However, complication by postembolization syndrome, such as abdominal pain, fever, nausea, vomiting and liver enzyme elevation, developed in $20-80 \%$ after Lip-TACE [3, 4], whereas symptoms developed in only 18-37\% after DEB-TACE [5, 6], showing that the complications were milder than those after Lip-TACE. On the other hand, DEB-TACE induced liver abscesses at a relatively high frequency, which could be fatal $[5,7-$ 9]. Adverse events after DEB-TACE have been reported occasionally [5, 7-11], but as it has just been introduced in Japan, many points regarding adverse events are unclear with respect to the developmental mechanism and risk factors.

We encountered a HCC patient who underwent DEB-TACE and experienced delayed intratumoral hemorrhage. Herein, we report on this case and present a review of the literature.

\section{Case Report}

An 81-year-old male regularly visited his physician for treatment of hepatitis C virusrelated cirrhosis. On routine abdominal ultrasonography (US), a mass was detected in S5 of the liver. On esophagogastroduodenoscopy, a type 0-IIa early gastric carcinoma with a diameter of $25 \mathrm{~mm}$ was detected in the lesser curvature of the middle body. The patient was referred to our hospital for further examination and treatment. During the first examination, the liver function was classified as Child-Pugh class $\mathrm{A}$, and the tumor markers were normal (AFP level: $3.4 \mathrm{ng} / \mathrm{ml}$, AFP-L3 fraction level $<0.5 \%$ ), but the PIVKA II had a level of 118 $\mathrm{mAU} / \mathrm{ml}$, showing a mild elevation. The mass in S5 of the liver was $35 \mathrm{~mm}$ in diameter. It was capsulated and enhanced in the arterial phase on dynamic computed tomography (CT) (fig. 1), and showed a mosaic pattern accompanied by a marginal hypoechoic zone on grayscale US. The mass was enhanced in the vascular phase $(0-40 \mathrm{~s})$ on contrast-enhanced US (CEUS) using Sonazoid ${ }^{\circledR}$ (Daiichi Sankyo, Tokyo, Japan), and showed a hypoechoic area in the postvascular phase (10 min after an intravenous injection of Sonazoid). Based on the tumor marker and imaging findings, the patient was diagnosed with HCC. DEB-TACE was performed because the patient declined to undergo surgical resection of HCC.

Access for TACE was performed under sterile conditions and under local anesthesia, via the right femoral artery using a 3-Fr sheath (Medikit, Tokyo, Japan) and in a retrograde fashion. An intensely stained tumor was detected in the S5 region on celiac arterial 
angiography. When a microcatheter was advanced to the anterior segmental artery of the right hepatic artery and contrast imaging was applied, an intensely stained tumor was observed, with A5 as the feeding artery. A solution impregnated with 100-300 $\mu \mathrm{m}$ DC Bead and $50 \mathrm{mg}$ epirubicin hydrochloride, with a DC Bead volume of $0.35 \mathrm{ml}$, was administered via the feeding artery to perform DEB-TACE. Disappearance of the intensely stained tumor image was confirmed by right hepatic arterial angiography, and the treatment was completed. The course after DEB-TACE was favorable, and no adverse events above grade 3 according to the Common Terminology Criteria for Adverse Events (CTCAE) version 4.0 were observed. Endoscopic submucosal dissection (ESD) of the early gastric carcinoma was performed about 1 month after DEB-TACE. Right hypochondriac pain suddenly developed 3 days after ESD, but no adverse events were assumed to be caused by ESD (i.e., free air, noted on dynamic CT). However, the tumor diameter had increased from that before DEB-TACE, and the tumor showed a high-intensity area on unenhanced CT (fig. 2a), which was not enhanced in the arterial phase on dynamic CT, suggesting intratumoral hemorrhage. The hemodynamics of the tumor were followed using CEUS over the time before and after DEBTACE. On CEUS carried out on the day after DEB-TACE, the whole intratumoral enhancement decreased, and nonenhanced patchy regions, assumed to be necrosis, were noted in the tumor in the vascular phase. On CEUS performed 4 weeks after DEB-TACE, i.e., immediately before ESD, the tumor size was $33 \times 31 \mathrm{~mm}$, and the enhancement area was increased in the vascular phase (fig. 3) compared with the CEUS imaging of the day after DEB-TACE. However, the tumor had enlarged to $41 \times 36 \mathrm{~mm}$ on CEUS when the right hypochondriac pain developed ( 5 weeks after DEB-TACE), and changes in the echogenicity on gray-scale US and nonenhancement of almost the entire tumor in the vascular phase on CEUS were noted (fig. 2b). Based on the above findings, the cause of right hypochondriac pain may have been DEB-TACE-associated intratumoral hemorrhage. Since the hemorrhage was limited to inside the tumor, it stopped spontaneously with rest.

\section{Discussion}

The advantages of DEB-TACE are as follows: the infusion dose of the chemotherapeutic agent can be modified by adjusting the chemotherapeutic agent carrier, while altering the depth of embolization with beads, the duration of tumor exposure to chemotherapeutic agent can be fine-tuned through sustained release of the chemotherapeutic agent, and systemic adverse reactions of chemotherapeutic agent can be attenuated by continuous release of high-dose chemotherapeutic agents into the tumors [12]. DEB has proven to be extremely potent and safe by allowing large amounts of the chemotherapeutic agent to concentrate within the tumor over time, thereby maximizing the cytotoxic effect, which also makes it attractive as an effective treatment for large lesions [11]. Favorable therapeutic effects have been reported occasionally $[8,9]$ and have increased the expectations of HCC treatment with DEB-TACE; however, the beads exhibit characteristic physical properties and behavior that are different from those of gelatin particles. It is important to fully investigate these characteristics to avoid inadequacy of the therapeutic effect and to prevent the onset of adverse events.

Previous studies have reported that the incidence of mild adverse events, such as postembolization syndrome, is lower in the DEB-TACE group compared with the Lip-TACE group [3-6]. Meanwhile, liver abscesses were induced at a relatively high frequency (about 1.6-7.4\%) [5, 7-9]. Malagari et al. [7] reported that the 30-day mortality was $1.26 \%$ among 237 HCC patients treated with DEB-TACE with DC Bead loaded with doxorubicin, and that 
the cause was liver abscess in all cases. Regarding other adverse events, acute pancreatitis and cholecystitis developed with incidences of $1-5$ and 1-6.3\%, respectively. Previous studies have shown that rare adverse events included liver failure and infection, bile duct injury, upper gastrointestinal bleeding, pulmonary embolism, splenic infarction, or spinal embolization $[1,7,10,11]$. Unlike Lip-TACE, many adverse events occurred due to ischemic changes induced by the inflow of beads into organs other than the HCC lesion, which is characteristic of DEB-TACE.

Tumor hemorrhage has also been reported after DEB-TACE $[1,7,10,11,13]$ with large tumors, with a diameter of 5-16 cm, and the tumor locations were subcapsular in all previous reports. To our knowledge, there has been no case of a tumor with a diameter $<5$ $\mathrm{cm}$ distinct from the subcapsular, as was observed in our patient.

There has been no report of a mechanism underlying the DEB-TACE-specific tumor hemorrhage. In general, the mechanism of tumor rupture after TACE might be related to increased intratumoral pressure as a result of rapid edematic expansion due to tumor necrosis or vascular injury, secondary to embolization [14]. The risk is particularly high in subcapsular lesions, and we must be careful because the spread of hemorrhage in the abdominal cavity cannot only lead to hemorrhagic shock, but also peritoneal metastasis. Unlike the reported cases of tumor hemorrhage after DEB-TACE, in the present case, the tumor diameter was not large and the tumor was distinct from the subcapsular region. However, intratumoral hemorrhage occurred about 1 month after DEB-TACE. Local pooling of the contrast agent within the target tumor could occur during DEB-TACE, and Seki et al. [10] reported that it occurred in $25.2 \%$ of 135 HCC patients treated with DEB-TACE (epirubicin-loaded HepaSphere). While the cause of this local pooling remains unclear, it is similar to extravasation or blood sinus [15], suggesting that it was a local hemorrhage due to intratumor vascular collapse as a consequence of the treatment. Injected beads can easily escape into the pooling space. When local pooling of the contrast agent was noted, Seki et al. [10] added a small amount of gelatin sponge particles, with a diameter ranging from 1 to 2 $\mathrm{mm}$, to the feeding artery until the pooling disappeared. Based on the above findings, the observation of local pooling of the contrast agent within the target tumor during treatment suggests that intratumoral pressure has already increased, which was unlikely in our patient. Treatment-induced intratumoral hemorrhage continued immediately after DEBTACE because no pooling was noted during treatment or on confirmation angiography following treatment. We considered that embolization was sufficient during treatment. However, it is possible that the amount of DC Bead was insufficient and embolization was incomplete. It was assumed that incomplete embolization subsequently caused intratumoral vascular collapse, i.e., a phenomenon similar to pooling, and that the hemorrhage expanded to the necrotic intratumoral area by sustained release of the chemotherapeutic agent and embolization, leading to complication of intratumoral hemorrhage despite the low risk of tumor hemorrhage.

DEB-TACE has just been introduced in Japan. Many points remain unclear, i.e., the appropriate bead sizes for each case and the level of embolization, but it may be important to apply sufficient embolization because insufficient embolization is likely to lead to tumor hemorrhage. DEB-TACE is considered to rarely cause adverse events, but fatal complications have been reported [5, 7-9], and reports of adverse events may increase with augmentation of the numbers of DEB-TACE-treated patients. If reports of tumor hemorrhage after DEBTACE increase, the mechanism of intratumoral hemorrhage in cases similar to our patient may be elucidated.

Intratumoral hemorrhage was suspected due to the presence of a high-intensity area in the tumor on unenhanced CT and a rapid increase in the tumor diameter and accompanying 
enlargement of the unenhanced area on CEUS performed before and after DEB-TACE. Based on these findings, intratumoral hemorrhage was diagnosed. It was difficult to assess the therapeutic effect of DEB-TACE by CT, based on Lipiodol accumulation, unlike that after conventional Lip-TACE, and evaluation of the intratumoral hemodynamics could be important. CEUS is superior in evaluating intratumoral hemodynamics in real time, and the utility of CEUS to evaluate the therapeutic effect after DEB-TACE was suggested.

\section{Conclusion}

We encountered a patient who experienced intratumoral hemorrhage about 1 month after DEB-TACE. DEB-TACE for HCC is a new treatment method in Japan, and reports of adverse events may increase in the future. To obtain the therapeutic effect of DEB-TACE while preventing the adverse events, it may be important to understand the characteristics of the beads themselves and to apply the appropriate embolization to each individual case. It was also suggested that CEUS is useful to evaluate the therapeutic effect after DEB-TACE.

\section{References}

1 Poon RT, Tso WK, Pang RW, Ng KK, Woo R, Tai KS, Fan ST: A phase I/II trial of chemoembolization for hepatocellular carcinoma using a novel intra-arterial drug-eluting bead. Clin Gastroenterol Hepatol 2007;5:1100-1108.

2 Lammer J, Malagari K, Vogl T, Pilleul F, Denys A, Watkinson A, Pitton M, Sergent G, Pfammatter T, Terraz S, Benhamou Y, Avajon Y, Gruenberger T, Pomoni M, Langenberger H, Schuchmann M, Dumortier J, Mueller C, Chevallier P, Lencioni R; PRECISION V Investigators: Prospective randomized study of doxorubicin-elutingbead embolization in the treatment of hepatocellular carcinoma: results of the PRECISION V study. Cardiovasc Intervent Radiol 2010;33:41-52.

-3 Brown KT, Nevins AB, Getrajdman GI, Brody LA, Kurtz RC, Fong Y, Blumgart LH: Particle embolization for hepatocellular carcinoma. J Vasc Interv Radiol 1998;9:822-828.

-4 Chan AO, Yuen MF, Hui CK, Tso WK, Lai CL: A prospective study regarding the complications of transcatheter intraarterial lipiodol chemoembolization in patients with hepatocellular carcinoma. Cancer 2002;94:1747-1752.

5 Varela M, Real MI, Burrel M, Forner A, Sala M, Brunet M, Ayuso C, Castells L, Montana X, Llovet JM, Bruix J: Chemoembolization of hepatocellular carcinoma with drug eluting beads: efficacy and doxorubicin pharmacokinetics. J Hepatol 2007;46:474-481.

-6 Grosso M, Vignali C, Quaretti P, Nicolini A, Melchiorre F, Gallarato G, Bargellini I, Petruzzi P, Massa Saluzzo C, Crespi S, Sarti I: Transarterial chemoembolization for hepatocellular carcinoma with drug-eluting microspheres: preliminary results from an Italian multicentre study. Cardiovasc Intervent Radiol 2008;31:1141-1149.

7 Malagari K, Pomoni M, Spyridopoulos TN, Moschouris H, Kelekis A, Dourakis S, Alexopoulou E, Koskinas J, Angelopoulos M, Kornezos J, Pomoni A, Tandeles S, Marinis A, Rizos S, Kelekis D: Safety profile of sequential transcatheter chemoembolization with DC Bead ${ }^{\mathrm{TM}}$ : results of 237 hepatocellular carcinoma (HCC) patients. Cardiovasc Intervent Radiol 2011;34:774-785.

8 Malagari K, Pomoni M, Moshouris H, Bouma E, Koskinas J, Stefaniotou A, Marinis A, Kelekis A, Alexopoulou E, Chatziioannou A, Chatzimichael K, Dourakis S, Kelekis N, Rizos S, Kelekis D: Chemoembolization with doxorubicin-eluting beads for unresectable hepatocellular carcinoma: five-year survival analysis. Cardiovasc Intervent Radiol 2012;35:1119-1128.

-9 Burrel M, Reig M, Forner A, Barrufet M, de Lope CR, Tremosini S, Ayuso C, Llovet JM, Real MI, Bruix J: Survival of patients with hepatocellular carcinoma treated by transarterial chemoembolization (TACE) using Drug Eluting Beads. Implications for clinical practice and trial design. J Hepatol 2012;56:1330-1335.

10 Seki A, Hori S, Kobayashi K, Narumiya S: Transcatheter arterial chemoembolization with epirubicin-loaded superabsorbent polymer microspheres for 135 hepatocellular carcinoma patients: single-center experience. Cardiovasc Intervent Radiol 2011;34:557-565.

-11 Ritter CO, Wartenberg M, Mottok A, Steger U, Goltz JP, Hahn D, Kickuth R: Spontaneous liver rupture after treatment with drug-eluting beads. Cardiovasc Intervent Radiol 2012;35:198-202. 


\section{Case Reports in Oncology}

\begin{tabular}{l|l}
\hline Case Rep Oncol 2014;7:739-745 \\
\hline DOI: $10.1159 / 000369005$ & $\begin{array}{l}\text { C 2014 S. Karger AG, Basel } \\
\text { www.karger.com/cro }\end{array}$ \\
\hline
\end{tabular}

Shiozawa et al.: Delayed Intratumoral Hemorrhage after Drug-Eluting Bead Transarterial Chemoembolization for Hepatocellular Carcinoma

12 Jordan O, Denys A, De Baere T, Boulens N, Doelker E: Comparative study of chemoembolization loadable beads: in vitro drug release and physical properties of DC bead and hepasphere loaded with doxorubicin and irinotecan. J Vasc Interv Radiol 2010;21:1084-1090.

-13 Nawawi O, Hazman M, Abdullah B, Vijayananthan A, Manikam J, Mahadeva S, Goh K: Transarterial embolization of hepatocellular carcinoma with doxorubicin-eluting beads: single centre early experience. Biomed Imaging Interv J 2010;6:e7.

14 Liu CL, Ngan H, Lo CM, Fan ST: Ruptured hepatocellular carcinoma as a complication of transarterial oily chemoembolization. Br J Surg 1998;85:512-514.

15 Osuga K, Hori S, Hiraishi K, Sugiura T, Hata Y, Higashihara H, Maeda N, Tomoda K, Nakamura H: Bland embolization of hepatocellular carcinoma using superabsorbent polymer microspheres. Cardiovasc Intervent Radiol 2008;31:1108-1116.

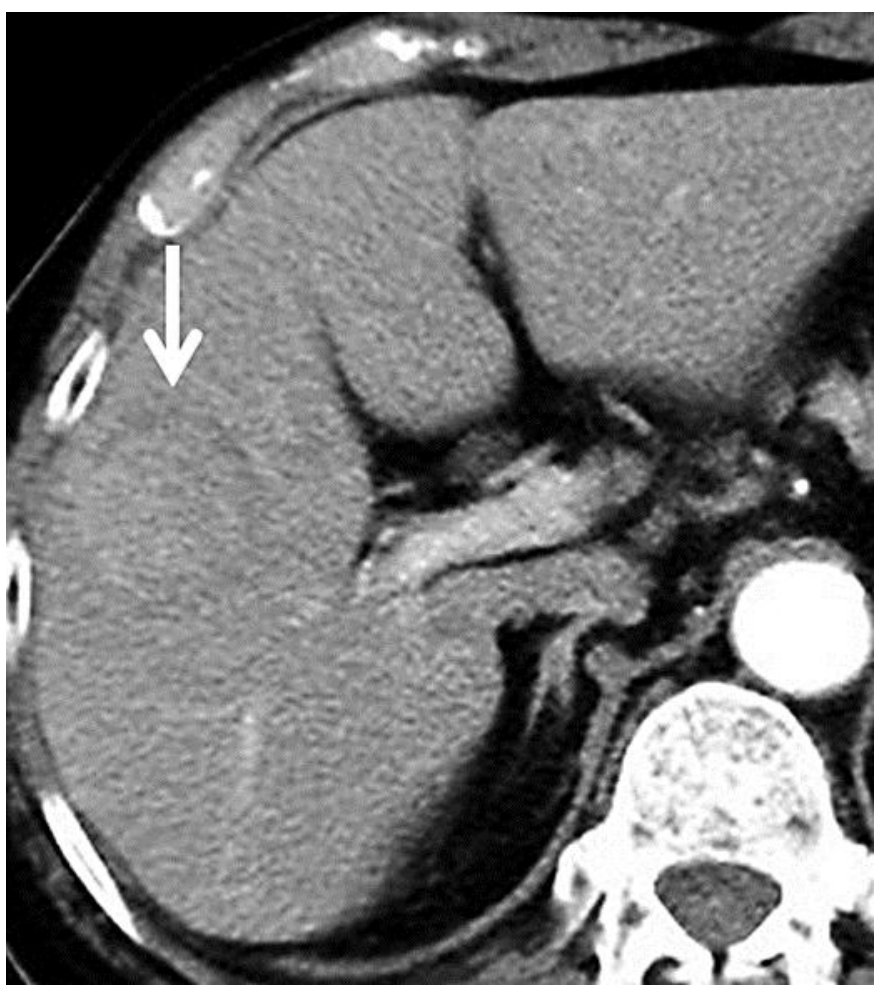

Fig. 1. Abdominal CT showing a slightly enhanced tumor of $35 \mathrm{~mm}$ in diameter in S5 of the liver in the arterial phase before DEB-TACE (arrow). 


\section{Case Reports in Oncology}

\begin{tabular}{l|l}
\hline Case Rep Oncol 2014;7:739-745 \\
\hline DOI: 10.1159/000369005 & $\begin{array}{l}\text { C 2014 S. Karger AG, Basel } \\
\text { www.karger.com/cro }\end{array}$ \\
\hline
\end{tabular}

Shiozawa et al.: Delayed Intratumoral Hemorrhage after Drug-Eluting Bead Transarterial Chemoembolization for Hepatocellular Carcinoma

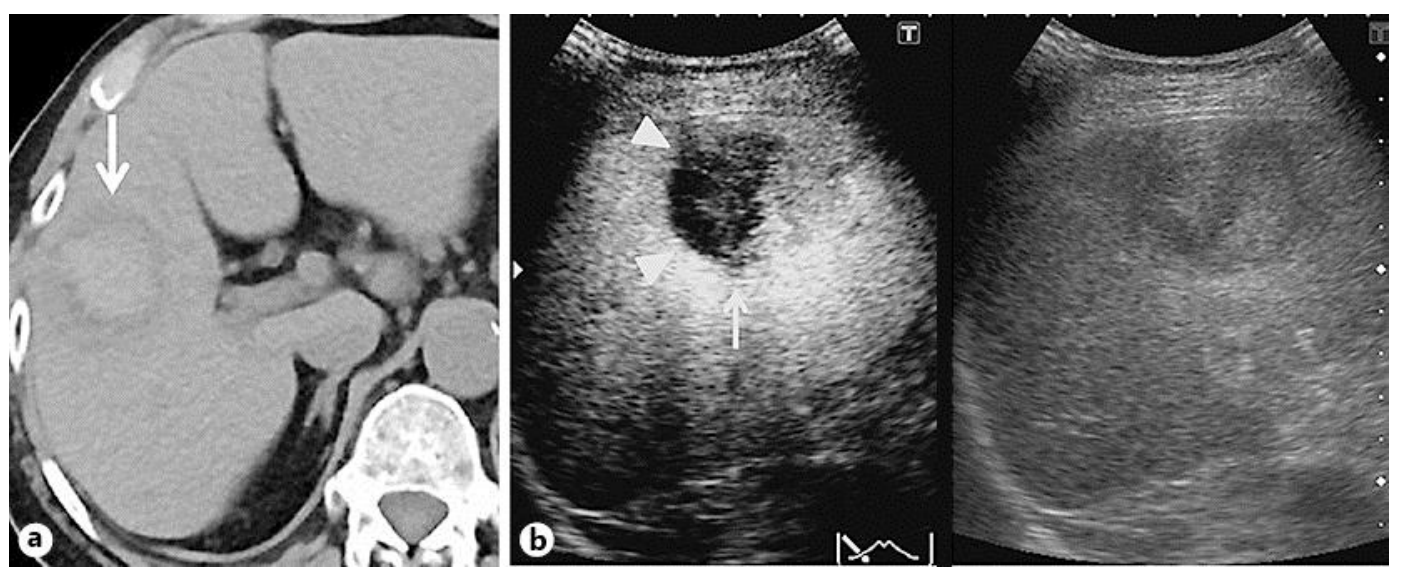

Fig. 2. a Unenhanced abdominal CT showing an increase of the tumor diameter and a high-intensity area in the tumor at the time of right hypochondriac pain (arrow). $\mathbf{b}$ Left panel: CEUS at the time of right hypochondriac pain shows a nonenhancement area (white arrowhead) of almost the entire tumor (arrow) in the vascular phase (40 s after injection of Sonazoid). Right panel: Gray-scale US (monitor mode).
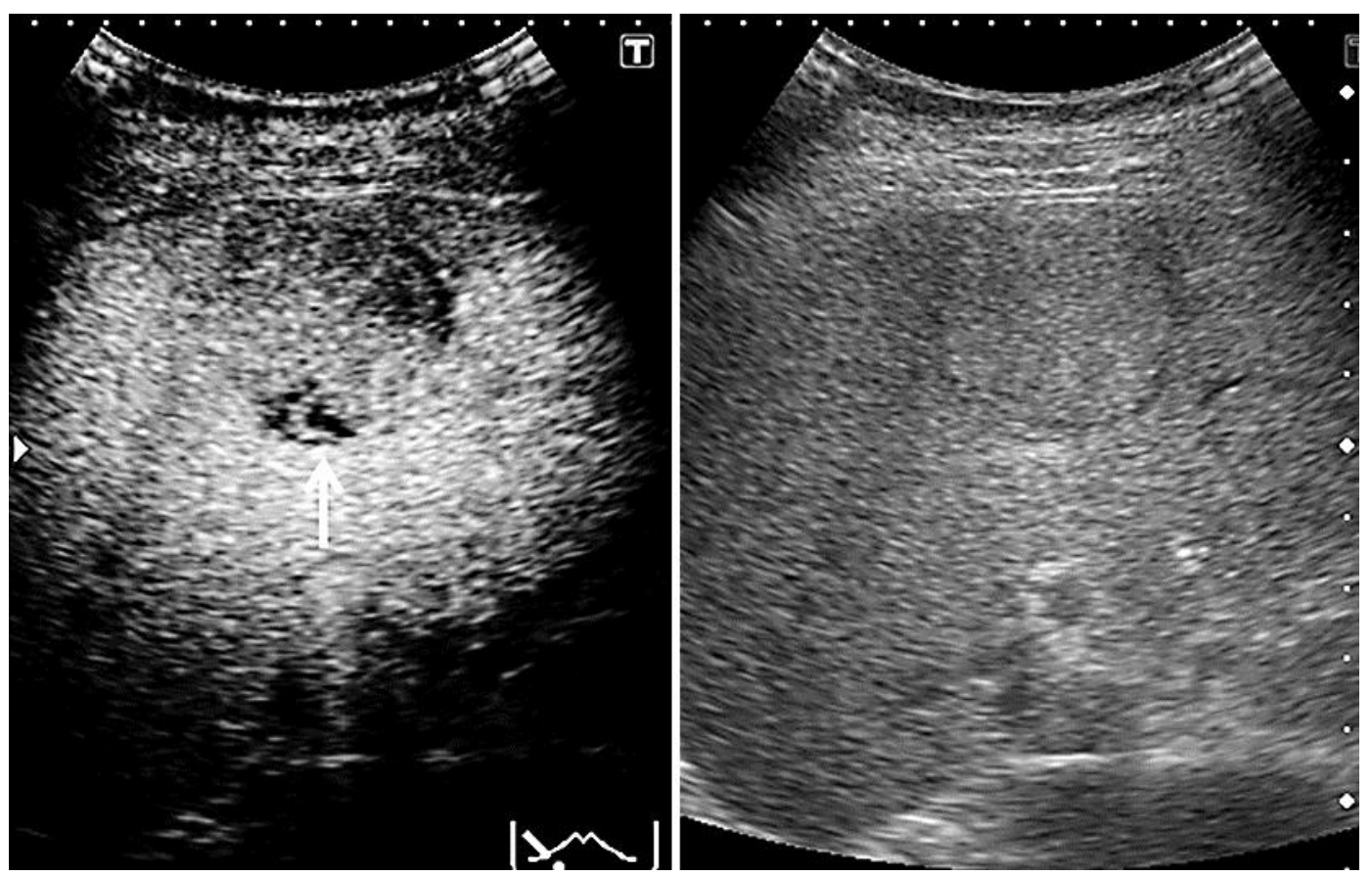

Fig. 3. Left panel: CEUS (40 s after injection of Sonazoid) about 1 month after DEB-TACE (3 days before the onset of right hypochondriac pain) shows an increase of the enhancement area in the vascular phase (arrow) compared with the CEUS imaging the day after DEB-TACE. Right panel: Gray-scale US (monitor mode). 